\title{
Correction to: GMR: graph-compatible MapReduce programming model
}

\author{
Weidong Zhang ${ }^{1}$ (D) Boxin $\mathrm{He}^{1} \cdot$ Yifeng Chen ${ }^{1} \cdot$ \\ Qifei Zhang ${ }^{2}$
}

Published online: 17 October 2017

(C) Springer Science+Business Media, LLC 2017

\section{Correction to: Multimed Tools Appl https://doi.org/10.1007/s11042-017-5102-2}

In the original publication, Fig. 12 was incorrectly presented. The plot line and legends of Fig. $12 \mathbf{a}, \mathbf{c}, \mathbf{e}$ and $\mathbf{f}$ should not overlap. The original article was corrected.

\author{
Weidong Zhang \\ zhangwd@pku.edu.cn \\ Boxin He \\ heboxin@pku.edu.cn \\ Yifeng Chen \\ cyf@pku.edu.cn \\ Qifei Zhang \\ zhangqf@cst.zju.edu.cn
}

1 Peking University, Beijing, China

2 Zhejiang University, Hangzhou, China 